\title{
Electro-fluid dynamics of aqueous humor production: simulations and new directions
}

Aurelio Giancarlo Mauri ${ }^{1}$, Lorenzo Sala ${ }^{2}$, Paolo Airoldi ${ }^{1}$, Giovanni Novielli ${ }^{3}$, Riccardo Sacco $^{1}$, Simone Cassani ${ }^{4}$, Giovanna Guidoboni ${ }^{4,2}$, Brent Siesky ${ }^{5}$, Alon Harris ${ }^{5}$

${ }^{1}$ Dipartimento di Matematica, Politecnico di Milano, Milano, Italy, ${ }^{2}$ Institut de Recherche Mathématique Avancée CNRS and Université de Strasbourg 7, Strasbourg Cedex, France, ${ }^{3}$ ST Microelectronics, Agrate Brianza (MB) Italy, ${ }^{4}$ Indiana University and Purdue University at Indianapolis, Department of Mathematical Sciences, IN, USA, ${ }^{5}$ Eugene and Marilyn Glick Eye Institute, Indiana University School of Medicine, IN, USA

\section{Abstract}

Purpose: to theoretically investigate the role of bicarbonate ion $\left(\mathrm{HCO}_{3}^{-}\right)$on the nonpigmented transepithelial potential difference $V_{m}$, the sodium potassium pump $(\mathrm{Na} / \mathrm{K})$ and the active secretion of aqueous humor.

Methods: a three-dimensional mathematical model is proposed to isolate the roles of $\mathrm{HCO}_{3}^{-}$and $\mathrm{Na}^{+}$, which are difficult to investigate experimentally. The model combines the velocity-extended Poisson-Nernst-Planck equations to describe ion electrodiffusion and the Stokes equations to describe aqueous humor flow into the basolateral space adjacent to the nonpigmented ephitelial cells.

Results: Computations show that $V_{m}$ is close to baseline experimental measurements (on monkeys) in the range $[-2.7,-2.3] \mathrm{mV}$ only if $\mathrm{HCO}_{3}^{-}$is included in the simulation. The model is also capable of reproducing the flow of $\mathrm{Na}^{+}$exiting the cell and the flow of $\mathrm{K}^{+}$entering the cell, in accordance with the physiology of the $\mathrm{Na} / \mathrm{K}$ pump. The simulated $\mathrm{Na} / \mathrm{K}$ ratio is 1.53 , which is in very good agreement with the theoretical value of 1.5.

Conclusion: Model simulations suggest that $\mathrm{HCO}_{3}^{-}$inhibition may prevent physiologically correct baseline values of the nonpigmented transepithelial potential difference and $\mathrm{Na} / \mathrm{K}$ ATPase function. This may provide useful indication in the design of medications that decrease the active secretion of aqueous humor, and supports the

Correspondence: Aurelio Giancarlo Mauri, Dipartimento di Matematica, Politecnico di Milano, Piazza Leonardo da Vinci 32, 20133 Milano, Italy.

E-mail: aureliogiancarlo.mauri@polimi.it 
advantage of using mathematical models as a noninvasive complement of animal models.

Keywords: aqueous humor production, electrochemical transport, mathematical modeling, numerical simulation, sodium-potassium pump

Abbreviations/Nomenclature: $\mathrm{HCO}_{3}^{-}$: bicarbonate; $\mathrm{Na}^{+}$: sodium; $\mathrm{K}^{+}$: potassium; $\mathrm{Cl}^{-}$: chloride; ATPase: adenosine triphosphatase; IOP: intraocular pressure; AH: aqueous humor; VE-PNP: velocity-extended Poisson-Nernst-Planck; NPE: nonpigmented epithelial cell; PDE: partial differential equation.

\section{Introduction}

Elevated intraocular pressure (IOP) is a recognized risk factor for glaucoma. Evidences show that the steady-state value of IOP is determined by the balance between production and drainage of aqueous humor (AH), and that sodium $\left(\mathrm{Na}^{+}\right)$and bicarbonate $\left(\mathrm{HCO}_{3}^{-}\right)$secretion into the basolateral space between nonpigmented epithelial cells (NPEs) contribute to $\mathrm{AH}$ production (see ${ }^{1}$ and references cited therein). A possible strategy to reduce IOP via topical medications is to reduce AH production via betaantagonists, alpha-agonists and carbonic anhydrase inhibitors ${ }^{2,3}$. The connection between $\mathrm{HCO}_{3}^{-}, \mathrm{Na}^{+}$and topical medications in the regulation of $\mathrm{AH}$ production is still controversial and difficult to study experimentally. Thus, the development and use of a mathematical model appears to be a promising approach to help unravel such a connection through simulation and comparison of different predicted scenarios.

In the present work, we propose a mathematical model capable of describing the relationship between nonpigmented epithelial transmembrane ion flow and $\mathrm{AH}$ production. The velocity-extended Poisson-Nernst-Planck (VE-PNP) equations are used to describe ion electrodiffusion through a moving electrolyte medium, whereas the Stokes equations are used to describe the motion of the incompressible electrolyte fluid. The illustration and discussion of the theoretical properties and algorithms to numerically solve the coupled VE-PNP/Stokes system can be found $\mathrm{in}^{4-9}$. Here, the model is implemented within the three-dimensional object-oriented software platform MP-FEMOS (Multi-Physics Finite Element Modeling Oriented Simulator ${ }^{9-13}$ ).

The proposed model is validated against baseline values of the nonpigmented transepithelial potential difference $V_{m}$. Then, the model is used to predict ion current flow under different working conditions characterized by the absence or presence of bicarbonate ions in the electrolyte fluid. Model simulations suggest that $\mathrm{HCO}_{3}^{-}$inhibition may prevent to attain physiologically correct baseline values of $V_{m}$ and $\mathrm{Na} / \mathrm{K}$ ATPase function. 


\section{Methods}

AH production within the ciliary body is driven by three main mechanisms: (a) transport of aqueous components by the ciliary circulation; (b) ultrafiltration and diffusion of clear fluid across the capillary-stroma barrier, and (c) osmotic efflux of clear fluid into the posterior chamber ${ }^{3}$. In this paper, we focus on the osmotic efflux, which is due to the active secretion of ions into the basolateral space between nonpigmented epithelial cells. Interestingly, ion accumulation in the basolateral space generates a transepithelial potential difference $V_{m}$ measured experimentally in various animal species, as reported in Table 1.

Table 1. Reference data for the transepithelial potential difference $V_{m}$ for several animal species.

\begin{tabular}{|c|c|c|}
\hline$V_{m}[\mathrm{~m} V]$ & animal species & reference \\
\hline $3.80 \pm 0.26$ & ox & 14 \\
\hline $5.53 \pm 0.41$ & ox & 15 \\
\hline $3.83 \pm 0.16$ & rabbit & 15 \\
\hline$-3.7 \pm 0.3$ & toad & 16 \\
\hline$-1.2 \pm 0.1$ & rabbit & 17 \\
\hline$-1.35 \pm 0.08$ & dog & 18 \\
\hline$-2.5 \pm 0.2$ & monkey & 19 \\
\hline
\end{tabular}
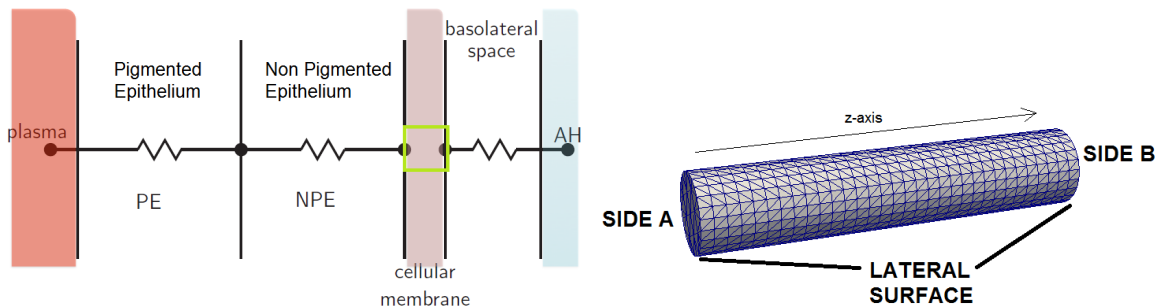

Fig. 1. Left panel: electrical scheme of the ciliary body system. PE: pigmented epithelial cellular layer. The various compartments are connected through resistances. Black bullets identify circuit nodes whose associated electrical variable is an electric voltage. The yellow box identifies the nonpigmented epithelial membrane region treated by the model. Right panel: geometry of a transmembrane channel in the yellow region. Side A represents the intracellular NPE region, Side $B$ represents the extracellular region in the basolateral space. The thickness of the channel is $5 \mathrm{~nm}$.

The resistive path between ciliary capillaries and posterior chamber is schematized in Fig. 1 (left panel). Based on the ionic concentrations reported in ${ }^{19}$ for the various segments in the path, it appears that the voltage drop between capillaries and 
NPE is 5 orders of magnitude smaller than the values in Tab. 1 so that, as a consequence, most of the transepithelial voltage drop occurs across the membrane of the NPE. Thus, in this work we focus on the NPE membrane, as indicated by the yellow square in Fig. 1 (left panel), and we consider a cylindrical ion channel whose inlet (SIDE A) and outlet (SIDE B) sections denote the intracellular NPE side and the basolateral space side, respectively, as depicted in Fig. 1 (right panel). We remark that the difference in potential between SIDE A and SIDE B corresponds to $V_{m}$. In particular, here we consider a particular type of ion channel, namely a sodium/potassium pump, since it has been proposed by many authors as important player in AH production ${ }^{1,3,20}$.

As mentioned in Section 1, we describe the electro-fluid dynamics within an ion channel in the NPE membrane by combining two main mathematical blocks:

- Velocity-Extended Poisson-Nernst-Planck (VE-PNP) equations to treat ionic transport into the basolateral space;

- Stokes equations to treat water movement down the resultant osmotic pressure gradient into the posterior chamber.

The VE-PNP equations express the conservation of each ionic charge moving across the ion channel and of the associated electrical current ${ }^{4}$. The Stokes equations express the conservation of mass and momentum of the electrolyte charged fluid flowing across the channel and transporting the ions at low Reynolds number ${ }^{4,5,7,8}$. The partial differential equations (PDEs) constituting the VE-PNP and the Stokes model blocks are illustrated in detail in Appendix A. The two blocks are mutually coupled through the volume force density due to the electric pressure exerted by the ions on each infinitesimal fluid element ${ }^{21}$. The solution of the VE-PNP/Stokes coupled system yields as output the spatio-temporal number density $n_{i}$ of the $i$-th ion species, $i=1, \ldots, M$, the electric potential $\varphi$, the electrolyte fluid velocity $\mathbf{u}$ and the fluid pressure $p$. Let $t$ denote the time variable, $t_{0}=0$ the starting simulation time and $T$ the total simulation time. The numerical approximation of the VE-PNP/Stokes coupled system is based on the following three steps:

1. We partition the time observational window $\left[t_{0}, t_{0}+T\right]$ into $N_{T} \geq 1$ time intervals $\tau_{k}=\left[t_{k}, t_{k+1}\right], k=0, \ldots, N_{T}-1$, of uniform width $\Delta t=T / N_{T}$;

2. For each $k=0, \ldots, N_{T}-1$, we advance in time using the Backward Euler time discretization method and we solve the PNP nonlinear block using a fixed point algorithm for a given velocity $\mathbf{u}^{k}$. This step returns the VE-PNP solution pair $\left(n_{i}^{k+1}, \varphi^{k+1}\right), i=1, \ldots, M$, at the next time level;

3. We use $n_{i}^{k+1}, i=1, \ldots, M$, and $\varphi^{k+1}$ to compute the electric pressure exerted by the ions on the fluid and we solve the Stokes linear block. This step returns the Stokes solution pair $\left(\mathbf{u}^{k+1}, p^{k+1}\right)$ at the next time level.

Numerical experiments for the validation of the model and the solution map schematically illustrated above are reported and discussed $i^{9}$ and $^{22}$ in the study of several nanoscale ionic channels of biophysical interest. 


\section{Results and discussion}

In this section we use the model illustrated in Section 2 to investigate the role of the NPE transmembranal sodium-potassium pump on the osmotic efflux of AH into the basolateral space. Boundary conditions on the surface of the cylindrical domain of Fig. 1 (right panel) are enforced in such a way that: (i) the $\mathrm{K}^{+}$ions enter the NPE at the intracellular side (A); (ii) the $\mathrm{Na}^{+}$ions enter the basolateral space at the aqueous side (B), with the ratio $\mathrm{Na}^{+} / \mathrm{K}^{+}=3: 2$ as in physiological $\mathrm{Na} / \mathrm{K}$ ATPase; (iii) the electrostatic potential $\varphi$ is grounded at side A; and (iv) the electric field flux is zero at side $B$. The values of all model parameters and of the initial and boundary conditions for the VEPNP system are summarized in Table 2. The longitudinal dimension of the simulated ion channel is $5 \mathrm{~nm}$ whereas the radius of the channel is $0.4 \mathrm{~nm}$. The computer simulations with the MP-FEMOS software platform run on a (rather coarse) grid made of 2000 tetrahedral elements and 600 vertices, with about 3000 degrees of freedom for the VE-PNP system and more than 10000 degrees of freedom for the Stokes system. Two distinct sets of ions are considered in the description of ion electrodiffusion, the first set including the species $\mathrm{K}^{+}, \mathrm{Na}^{+}$and $\mathrm{Cl}^{-}(M=3)$, the second set including also the $\mathrm{HCO}_{3}^{-}$ion $(M=4)$. The baseline ion concentrations are set according to the values reported $\mathrm{in}^{19}$.
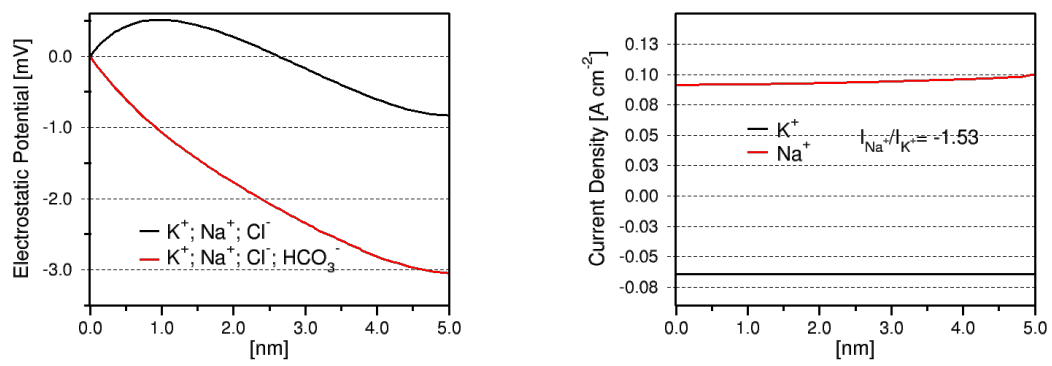

Fig. 2. Left panel: spatial distribution of $\varphi$ in the NPEC channel. Black curve: $\mathrm{HCO}_{3}^{-}$is inhibited. Red curve: $\mathrm{HCO}_{3}^{-}$is included. Right panel: spatial distributions of $\mathrm{K}^{+}$(black) and $\mathrm{Na}^{+}$(red) steady-state current densities.

Fig. 2 (left panel) illustrates the results of model validation against physiological reference data by plotting the steady-state spatial distribution of the electric potential (in $\mathrm{mV}$ ). The computed nonpigmented transepithelial potential difference, $V_{m, c}$, is equal to the value of $\varphi$ at side B. The negative value of $V_{m, c}$ indicates local net anion accumulation. We notice that $V_{m, c}$ is close to experimental baseline measurement (for monkeys) in the range $[-2.7,2.3] \mathrm{mV}^{19}$ (cf. Table 1, last row) only if bicarbonate is included in the simulation (red curve). Fig. 2 (right panel) illustrates model predictions showing the spatial distributions of the $\mathrm{Na}^{+}$and $\mathrm{K}^{+}$steady-state current den- 
sities (in $\mathrm{A} \mathrm{cm}^{-2}$ ). The positive value of the $\mathrm{Na}^{+}$current indicates that $\mathrm{Na}^{+}$ions exit the NPE whereas the negative value of the $\mathrm{K}^{+}$current indicates that $\mathrm{K}^{+}$ions enter the NPE, in accordance with the physiology of the $\mathrm{Na} / \mathrm{K}$ pump. The ratio between the (module) of the two currents is 1.53 in very good agreement with the theoretical value of 1.5 .

\section{Conclusions and future perspectives}

In the present article we have carried out the theoretical study of the osmotic efflux of aqueous humor into the posterior chamber of the eye due to the active secretion of ions into the basolateral space between nonpigmented epithelial cells. The research is motivated by the fact that it is rather difficult in experimental investigations to isolate the role of a single electrolyte in regulating aqueous humor production. To this purpose, we have devised a mathematical model localized at the nonpigmented transepithelial cellular scale with the aim of describing the ionic flux of sodium, potassium, chloride and bicarbonate across a single membrane ion channel to the basolateral space which is believed to lead ultimately to a net $\mathrm{H}_{2} \mathrm{O}$ efflux into the posterior eye chamber. The model is constituted by a system of partial differential equations representing balance laws for ionic charge, fluid mass, ionic momentum density, fluid momentum, mobile charge and electric field at each spatial position in the channel and at each time level. Simulation results tested against experimental measurements in monkeys suggest that bicarbonate inhibition may prevent to attain physiologically correct baseline values of the transepithelial membrane potential. Simulations accounting the presence of bicarbonate also predict the possibility of reproducing a physiologically correct $\mathrm{Na} / \mathrm{K}$ ATPase function. These results may provide useful indication in the design of IOP lowering medications to decrease aqueous humor production on an effective patient-specific basis, and support the advantage of using mathematical modelling as a noninvasive complement of the animal model. Future effort will be devoted to investigating the role of permanent surface charge and of protein conformation ${ }^{23}$ on the modulation of the electrophysiological response of the transepithelial membrane channel.

\section{Acknowledgements}

G. Guidoboni and S. Cassani were partially supported by the National Science Foundation with grants NSF-DMS 1134731 and 1224195, by the chair Gutenberg funds of the Cercle Gutenberg of the Region Alsace (France) and by LABEX IRMIA at the University of Strasbourg (France). A. Harris and B. Siesky were partially supported by National Institutes of Health Grant 1R21EY02210101A1, unrestricted grant from Research to Prevent Blindness, Inc. 
Table 2. Model parameters, boundary and initial conditions for the VE-PNP model. All values are taken as in ${ }^{22}$.

\begin{tabular}{|c|c|c|}
\hline Parameter & value & units \\
\hline$z_{\mathrm{K}^{+}}$ & +1 & {$[-]$} \\
\hline$z_{\mathrm{Na}^{+}}$ & +1 & {$[-]$} \\
\hline$z_{\mathrm{Cl}^{-}}$ & -1 & {$[-]$} \\
\hline$z_{\mathrm{HCO}_{3}^{-}}$ & -1 & {$[-]$} \\
\hline$\mu_{\mathrm{K}^{+}}$ & $1.957 \cdot 10^{-5}$ & $\mathrm{~cm}^{2} \mathrm{~V}^{-1} \mathrm{~s}^{-1}$ \\
\hline$\mu_{\mathrm{Na}^{+}}$ & $1.334 \cdot 10^{-5}$ & $\mathrm{~cm}^{2} \mathrm{~V}^{-1} \mathrm{~s}^{-1}$ \\
\hline$\mu_{\mathrm{Cl}^{-}}$ & $2.033 \cdot 10^{-5}$ & $\mathrm{~cm}^{2} \mathrm{~V}^{-1} \mathrm{~s}^{-1}$ \\
\hline$\mu_{\mathrm{HCO}_{3}^{-}}$ & $1.185 \cdot 10^{-5}$ & $\mathrm{~cm}^{2} \mathrm{~V}^{-1} \mathrm{~s}^{-1}$ \\
\hline $\bar{f}_{\mathrm{K}}+\left.\right|_{\text {SIDE A }}$ & $2 \cdot 10^{19}$ & $\mathrm{~cm}^{-2} \mathrm{~s}^{-1}$ \\
\hline $\bar{n}_{\mathrm{Na}}+\left.\right|_{\text {SIDE A }}$ & $7.82 \cdot 10^{19}$ & $\mathrm{~cm}^{-3}$ \\
\hline$\left.\bar{n}_{\mathrm{Cl}}\right|_{\text {SIDE A }}$ & $6.44 \cdot 10^{19}$ & $\mathrm{~cm}^{-3}$ \\
\hline $\bar{n}_{\mathrm{HCO}_{3}^{-}} \mid$SIDE A & $1.81 \cdot 10^{19}$ & $\mathrm{~cm}^{-3}$ \\
\hline$\left.\bar{n}_{\mathrm{K}^{+}}\right|_{\text {SIDE B }}$ & $2.41 \cdot 10^{18}$ & $\mathrm{~cm}^{-3}$ \\
\hline$\left.\bar{f}_{\mathrm{Na}^{+}}\right|_{\text {SIDE B }}$ & $3 \cdot 10^{19}$ & $\mathrm{~cm}^{-2} \mathrm{~s}^{-1}$ \\
\hline$\left.\bar{n}_{\mathrm{Cl}}{ }\right|_{\text {SIDE B }}$ & $7.89 \cdot 10^{19}$ & $\mathrm{~cm}^{-3}$ \\
\hline$\left.\bar{n}_{\mathrm{HCO}_{3}^{-}}\right|_{\text {SIDE B }}$ & $1.2 \cdot 10^{19}$ & $\mathrm{~cm}^{-3}$ \\
\hline$n_{\mathrm{K}^{+}}^{0}$ & $2.41 \cdot 10^{18}$ & $\mathrm{~cm}^{-3}$ \\
\hline$n_{\mathrm{Na}^{+}}^{0}$ & $8.19 \cdot 10^{19}$ & $\mathrm{~cm}^{-3}$ \\
\hline$n_{\mathrm{Cl}^{-}}^{0}$ & $7.17 \cdot 10^{19}$ & $\mathrm{~cm}^{-3}$ \\
\hline$n_{\mathrm{HCO}_{3}^{-}}^{0}$ & $1.51 \cdot 10^{19}$ & $\mathrm{~cm}^{-3}$ \\
\hline$T$ & 300 & $\mathrm{~K}$ \\
\hline$\epsilon_{f}$ & $708.32 \cdot 10^{-10}$ & $\mathrm{~F} \mathrm{~cm}^{-1}$ \\
\hline$\left.\overline{\bar{\varphi}}\right|_{\text {SIDE A }}$ & 0 & $\mathrm{~V}$ \\
\hline$\sigma_{\text {fixed }}$ & 0 & $\mathrm{C} \mathrm{cm}^{-2}$ \\
\hline$\mu_{f}$ & $10^{-2}$ & $\mathrm{~g} \mathrm{~cm}^{-1} \mathrm{~s}^{-1}$ \\
\hline$\rho_{f}$ & 1 & $\mathrm{~g} \mathrm{~cm}^{-3}$ \\
\hline $\mathrm{g}$ & $\mathbf{0}$ & $\mathrm{cm} \mathrm{s}^{-1}$ \\
\hline $\mathbf{u}^{0}$ & 0 & $\mathrm{~cm} \mathrm{~s}^{-1}$ \\
\hline$\left.\bar{p}\right|_{\text {SIDE A }}$ & 0 & $\mathrm{~g} \mathrm{~cm}^{-1} \mathrm{~s}^{-2}$ \\
\hline$\left.\bar{p}\right|_{\text {SIDE B }}$ & 0 & $\mathrm{~g} \mathrm{~cm}^{-1} \mathrm{~s}^{-2}$ \\
\hline
\end{tabular}




\section{A. Mathematical Model}

In Sect. A.1 we introduce the mathematical description of the problem geometry. In Sect. A.2 we describe the PNP equations for ion flow and in Sect. A.3 we describe the Stokes equations for the intrachannel electrolyte fluid.

\section{A.1 Geometry}

Let $\mathbf{x}$ and $t$ denote the spatial and temporal coordinates, respectively. The simulation domain $\Omega \subset \mathbb{R}^{3}$ (the ion channel) is shown in Fig. 1 (right panel). The channel is filled with an incompressible, viscous and Newtonian fluid moving with velocity $\mathbf{u}=$ $\mathbf{u}(\mathbf{x}, t)$, in which $M \geq 1$ chemical species are dissolved. Each chemical has effective charge $z_{i}, i=1, \ldots, M$, and concentration (number density) $n_{i}=n_{i}(\mathbf{x}, t)\left[\mathrm{cm}^{-3}\right]$. Cations have $z_{i}>0$, anions have $z_{i}<0$. We denote by $\partial \Omega$ the boundary of the domain $\Omega$ and by $\mathbf{n}$ the unit outward normal vector on $\partial \Omega$. With each dependent variable $U$, we associate a partition of the domain boundary $\partial \Omega$ into the union of (generally different) subsets. We indicate by $\Gamma_{D}^{U}$ the subset of $\partial \Omega$ where a Dirichlet condition and by $\Gamma_{N}^{U}$ the subset where a Neumann condition is applied, in such a way that $\Gamma_{D}^{U} \cup \Gamma_{N}^{U}=\partial \Omega$ and $\Gamma_{D}^{U} \cap \Gamma_{N}^{U}=\varnothing$.

\section{A.2 The Velocity-Extended Poisson-Nernst-Planck equation system}

The Velocity-Extended Poisson-Nernst-Planck system to describe the electrodiffusion of $M$ ion species $n_{i}, i=1, \ldots, M$, throughout a fluid in motion with velocity $\mathbf{u}$ consists of the following PDEs:

$$
\begin{aligned}
& \frac{\partial n_{i}}{\partial t}+\operatorname{div} \mathbf{f}_{i}=0, \\
& \mathbf{f}_{\mathbf{i}}=\frac{z_{i}}{\left|z_{i}\right|} \mu_{i} n_{i} \mathbf{E}-D_{i} \nabla n_{i}+n_{i} \mathbf{u}, \\
& \operatorname{div} \mathbf{D}=q \sum_{i=1}^{M} z_{i} n_{i} \\
& \mathbf{D}=\epsilon_{f} \mathbf{E}=-\epsilon_{f} \nabla \varphi
\end{aligned}
$$

We refer to ${ }^{4,24-26}$ for a detailed derivation of (1), to $0^{5,7}$ for the study of existence of its solution and to ${ }^{11,27}$ for recent applications to ion channel simulations. In (1), the symbol $\mathbf{f}_{i}$ denotes the ion particle flux $\left[\mathrm{cm}^{-2} \mathrm{~s}^{-1}\right], \mu_{i}$ and $D_{i}$ are the ion electrical mobility and diffusivity and $T$ is system temperature. The third term in the right-hand side of the linear momentum balance equation (1b) expresses the coupling between the motion of the ion species and the moving electrolyte fluid. In the Poisson equation (1c), D is the electric displacement, $\mathbf{E}$ and $\varphi$ are the electric field $\left[\mathrm{Vcm}^{-1}\right]$ and electric potential [V], respectively, whereas $q=1.602 \cdot 10^{-19}[\mathrm{C}]$ and $\epsilon_{f}$ denote the electron charge and the electrolyte fluid dielectric permittivity, respectively. The diffusion coefficient 
$D_{i}$ and the mobility $\mu_{i}$ are proportional through the Einstein relation

$$
D_{i}=\mu_{i} \frac{k_{B} T}{q\left|z_{i}\right|}
$$

where $k_{B}=1.38 \cdot 10^{-16}\left[\mathrm{~cm}^{2} \mathrm{~g} \mathrm{~s}^{-2} \mathrm{~K}^{-1}\right]$ is Boltzmann's constant and $T$ is system temperature. Initial conditions for ion concentrations are $\forall i=1, \ldots, M$ :

$$
n_{i}(\mathbf{x}, 0)=n_{i}^{0}(\mathbf{x}) \quad \text { in } \Omega,
$$

where the functions $n_{i}^{0}$ are positive given data. The boundary conditions for the VEPNP system are $\forall i=1, \ldots, M$ :

$$
\begin{array}{ll}
\varphi=\bar{\varphi} & \text { on } \Gamma_{D}^{\varphi}, \\
\mathbf{D} \cdot \mathbf{n}=\sigma_{\text {fixed }} & \text { on } \Gamma_{N}^{\varphi}, \\
n_{i}=\bar{n}_{i} & \text { on } \Gamma_{D}^{n_{i}}, \\
\mathbf{f}_{\mathbf{i}} \cdot \mathbf{n}=\bar{f}_{i} & \text { on } \Gamma_{N}^{n_{i}},
\end{array}
$$

where $\bar{\varphi}$ is the electrostatic potential of the side $\Gamma_{D}^{\varphi}, \sigma_{\text {fixed }}\left[\mathrm{C} \mathrm{cm}^{-2}\right]$ is a fixed surface charge density on side $\Gamma_{N}^{\varphi}$ due to the lipid membrane bilayer and $\bar{n}_{i}$ is a given concentration of the chemical species $i$ on side $\Gamma_{D}^{n_{i}}$. Conditions (4a) and (4c) enforce a given voltage and a given concentration on the associated side, respectively, whereas (4b) and (4d) enforce nonhomogeneous Neumann conditions on each associated side.

\section{A.3 The Stokes equation system}

The Stokes system describing the slow motion of an incompressible viscous fluid with a constant density $\rho_{f}\left[\mathrm{~g} \mathrm{~cm}^{-3}\right]$ consists of the following PDEs:

$$
\begin{aligned}
& \operatorname{div} \mathbf{u}=0, \\
& \rho_{f} \frac{\partial \mathbf{u}}{\partial t}=\operatorname{div} \underline{\underline{\sigma}}(\mathbf{u}, p)+q \sum_{i=1}^{M} z_{i} n_{i} \mathbf{E}, \\
& \underline{\underline{\sigma}}(\mathbf{u}, p)=2 \mu_{f} \underline{\underline{\epsilon}}(\mathbf{u})-p \underline{\underline{\delta}}, \\
& \underline{\underline{\epsilon}}(\mathbf{u})=\underline{\underline{\nabla}}_{s} \mathbf{u}=\frac{1}{2}\left(\nabla \mathbf{u}+(\nabla \mathbf{u})^{T}\right) .
\end{aligned}
$$

We refer to ${ }^{28}$ for a complete mathematical and numerical treatment of the Stokes and Navier-Stokes equations for incompressible and compressible fluids. In (5), u is the fluid velocity $\left[\mathrm{cm} \mathrm{s}^{-1}\right], p$ the fluid pressure $[\mathrm{Pa}], \mu_{f}$ the fluid shear viscosity $\left[\mathrm{g} \mathrm{m}^{-1} \mathrm{~s}^{-1}\right], \underline{\underline{\sigma}}$ the stress tensor $\left[\mathrm{g} \mathrm{cm}^{-1} \mathrm{~s}^{-2}\right.$ ] and $\underline{\underline{\epsilon}}$ the strain rate tensor $\left[\mathrm{s}^{-1}\right]$. The symbol $\underline{\bar{\delta}}$ is the second-order identity tensor of dimension 3 and the second-order tensor $\underline{\bar{\nabla}}_{s} \mathbf{u}$ is the symmetric part of the gradient of $\mathbf{u}$. Notice that in accordance 
with the assumption of slow fluid motion, the quadratic convective term in the inertial forces has been neglected in the momentum balance equation (5b). The second term in the right-hand side of the momentum balance equation (5b) physically corresponds to the electric pressure exerted by the ionic charge on the electrolyte fluid, and mathematically represents the coupling between electrodiffusive ion transport and electrolyte fluid motion. The initial condition for electrolyte fluid velocity is

$$
\mathbf{u}(\mathbf{x}, 0)=\mathbf{u}^{0}(\mathbf{x}) \quad \text { in } \Omega,
$$

where the function $\mathbf{u}^{0}$ is a given datum, usually set equal to zero. The boundary conditions for the Stokes system are:

$$
\begin{array}{ll}
\mathbf{u}=\mathbf{g} & \text { on } \Gamma_{D}^{\mathbf{u}}, \\
\underline{\underline{\sigma}}(\mathbf{u}, p) \mathbf{n}=\mathbf{h} & \text { on } \Gamma_{N}^{\mathbf{u}},
\end{array}
$$

where $\mathbf{g}$ is the imposed velocity on the side $\Gamma_{D}^{\mathbf{u}}$ and $\mathbf{h}=-\bar{p} \mathbf{n}$ is the value of the traction on $\Gamma_{N}^{\mathbf{u}}$. Relation (7a) is the wall adherence condition (typically $\mathrm{g}=\mathbf{0}$ ) and $(7 \mathrm{~b})$ is the application of the action-reaction principle at the boundary.

\section{References}

1. Kiel JW. Physiology of the intraocular pressure. Pathophysiology of the Eye. Ed. by J Feher. Glaucoma (4): Akademiai Kiadò, Budapest, 1998; 79-107.

2. Gupta S, Niranjan D, Agrawal S, Srivastava S, Saxena R. Recent advances in pharmacotherapy of glaucoma. Indian Journal of Pharmacology, 2008;40(5): 197-208.

3. Kiel JW, Hollingsworth M, Rao R, Chen M, Reitsamer HA. Ciliary blood flow and aqueous humor production. Progress in Retinal and Eye Research, 2011;30(1): 1-17.

4. Rubinstein I. Electrodiffusion of Ions. SIAM, 1990;

5. Jerome JW. Analytical approaches to charge transport in a moving medium. Transport Theory and Statistical Physics, 2002;31, 333-366.

6. Longaretti M, Chini B, Jerome JW, Sacco R. Computational models in nano-bio-electronics: simulation of ionic transport in voltage operated channels. Journal of Nanoscience and Nanotechnology, 2007;8(7): 1-9.

7. Jerome JW, Sacco R. Global weak solutions for an incompressible charged fluid with multi-scale couplings: Initial-boundary value problem. Nonlinear Analysis, 2009;71, e2487-e2497.

8. Schmuck M. Analysis of the Navier-Stokes-Nernst-Planck-Poisson system. Mathematical Models and Methods in Applied Sciences, 2009;19(6): 993-1015.

9. Airoldi P, Mauri AG, Sacco R, Jerome JW. Three-dimensional numerical simulation of ion nanochannels. Journal of Coupled Systems and Multiscale Dynamics, 2015-04-01T00:00:00;3(1): 57-65.

10. Novielli G, Ghetti A, Varesi E, Mauri A, Sacco R. Atomic migration in phase change materials. 2013 IEEE International Electron Devices Meeting (IEDM) Washington, DC, USA. 2013; 22.3.1-22.3.4. doi: 10.1109/ IEDM.2013.6724683.

11. Mauri AG, Sacco R, Verri M. Electro-thermo-chemical computational models for $3 D$ heterogeneous semiconductor device simulation. Applied Mathematical Modelling, 2015;39(14): 4057 -4074.

12. Benvenuti A, Ghetti A, Mauri A, Liu H, Mouli C. Current and future prospects of non-volatile memory modeling. 2014 IEEE International Electron Devices Meeting (SISPAD) Yokohama, Japan. 2014; 249252. 
13. Mauri AG, Bortolossi A, Novielli G, Sacco R. 3D finite element modeling and simulation of industrial semiconductor devices including impact ionization. Journal of Mathematics in Industry, 2015;5(1): doi:10.1186/s13362-015-0015-z.

14. Cole DF. Electrical potential across the isolated ciliary body observed in vitro. Br J Ophthalmol, 1961;45(10): 641-653.

15. Cole DF. Electrical potential across the isolated ciliary body of ox and rabbit. Br J Ophthalmol, 1962;46(10): 577-591.

16. Watanabe T, Saito Y. Characteristics of ion transport across the isolated ciliary epithelium of the toad as studied by electrical measurements. Exp Eye Res, 1978;27(2): 215-226.

17. Krupin T, Reinach PS, Candia OA, Podos SM. Transepithelial electrical measurements on the isolated rabbit iris-ciliary body. Exp Eye Res, 1984;38(2): 115-123.

18. lizuka S, Kishida K, Tsuboi S, Emi K, Manabe R. Electrical characteristics of the isolated dog ciliary body. Curr Eye Res, 1984;3(3): 417-421.

19. Chu TC, Candia OA, Podos SM. Electrical parameters of the isolated monkey ciliary epithelium and effects of pharmacological agents. Invest Ophthalmol Vis Sci, 1987;28(10): 1644-1648.

20. Moses R. Intraocular pressure. Adler's Physiology of the Eye: Clinical Application. Ed. by R Moses, W Hart. C. V. Mosby Co., St Louis, 1987; 223-245.

21. Stratton J, Antennas I, Society P. Electromagnetic Theory. An IEEE Press classic reissue. Wiley, 2007;

22. Sacco R, Airoldi P, Mauri A, Jerome JW. Three-Dimensional Simulation of Biological lon Channels Under Mechanical, Thermal and Fluid Forces. ArXiv e-prints, 2015; arXiv: 1509.07301. Available from: http://adsabs.harvard.edu/abs/2015arXiv150907301S.

23. Malasics A, Gillespie D, Nonner W, Henderson D, Eisenberg B, Boda D. Protein structure and ionic selectivity in calcium channels: Selectivity filter size, not shape, matters. Biochimica et Biophysica Acta (BBA) - Biomembranes, 2009;1788(12): 2471 -2480.

24. Mori Y, Liu C, Eisenberg RS. A model of electrodiffusion and osmotic water flow and its energetic structure. Physica D: Nonlinear Phenomena, 2011;240(22): 1835-1852.

25. Ray N, Muntean A, Knabner P. Rigorous homogenization of a Stokes-Nernst-Planck-Poisson system. J. Math. Anal. Appl. 2012;390, 374-393.

26. Ray N, Noorden T van, Frank F, Knabner P. Multiscale Modeling of Colloid and Fluid Dynamics in Porous Media Including an Evolving Microstructure. Transport in Porous Media, 2012;95(3): 669-696.

27. Jerome JW, Chini B, Longaretti M, Sacco R. Computational modeling and simulation of complex systems in bio-electronics. Journal of Computational Electronics, 2008;7(1): 10-13.

28. Quarteroni A, Valli A. Numerical Approximation of Partial Differential Equations. Springer-Verlag, 1997; 